\title{
El uso de las TIC para promover la lectura en bibliotecas públicas con la intervención de los bibliotecarios
}

\author{
Sindy Catherine Rendón-Galvis* \\ Antonia Olivia Jarvio-Fernández**
}

Artículo recibido:

18 de marzo de 2019

Artículo aceptado:

9 de septiembre de 2019

Artículo de investigación

\section{Resumen}

El siglo XXI trae consigo una serie de transformaciones que impactan todos los ámbitos de la sociedad. Los procesos discursivos han evolucionado a formas más complejas y leer adquiere una importancia mayor al integrar nuevas expresiones comunicativas, como la multimodal. La enseñanza de la lectura requiere un cambio de metodología y contenido, reto que la educación tiene que afrontar sin delación. Las bibliotecas, como medios insustituibles para proporcionar información, requieren ajustar sus objetivos para coadyuvar en la tarea de la multialfabetización. La promoción de la lectura es una actividad consustancial a las tareas bibliotecarias y

* Universidad Veracruzana, México

cathezar15@gmail.com

** Centro de Estudios de la Cultura y la Comunicación de la Universidad Veracruzana, México ojarvio@uv.mx INVESTIGACIÓN BIBLIOTECOLÓGICA, vol.34, núm. 83, abril/junio, 2020, México, ISSN: 2448-8321 pp. 129-144 
la tecnología puede ser de gran valor para acercar a los usuarios a los textos. El presente trabajo presenta un proyecto de intervención de promoción de lectura utilizando las TIC, el cual fue realizado con un grupo de bibliotecarios de bibliotecas públicas. Se demuestra un impacto positivo del acompañamiento a las tareas de los bibliotecarios así como la necesidad de capacitación en promoción de la lectura utilizando las TIC.

Palabras clave: Bibliotecas Públicas; Trabajo Bibliotecario; Uso de Tecnología; Multimodalidad

The use of ICT to promote reading in public libraries with the intervention of librarians

Sindy Catherine Rendón-Galvis and Antonia Olivia Jarvio-Fernández

\begin{abstract}
The 21st century brings with it a series of transformations that impact all areas of society. Discursive processes have evolved to more complex forms and reading has acquired greater importance at integrating new communicative expressions, such as multimodality. The teaching of reading requires a change in methodology and content, a challenge that education has to face without delay. Libraries, as irreplaceable means to provide information, need to adjust their objectives for helping in the task of multiliteracy. The promotion of reading is a consubstantial activity to library tasks and technology can be of great value to approach users to the texts. This paper presents an intervention project to promote reading using ICT's, which was carried out with a library group of public libraries. This accompaniment showed a positive impact to the tasks of librarians as well as the need for training in reading promotion using ICT.
\end{abstract}

Keywords: Public Libraries; Library Work; Use of Technology; Multimodality 


\section{INTRODUCCIÓN}

T as tecnologías de la información y comunicación (TIC) han propiciado Luna revolución en la comunicación que ha permeado en la sociedad. Los dispositivos para la lectura han diversificado la manera como leemos y las formas de acceder a la información (Cassany, 2006 y 2012; Cordón García y Jarvio Fernández, 2015; Gómez Camacho, 2016). De igual manera la biblioteca se está transformando para atender los requerimientos de una sociedad digital, que requiere de apoyo para localizar información, usar adecuadamente el discurso multimodal y darle valor a lo que lee. En muchos países, las generaciones actuales se caracterizan por la deficiencia en competencias de lectoescritura, lo que redunda en inconvenientes para leer en los nuevos formatos; sólo utilizan el correo electrónico y redes sociales, la lectura es superficial y tienen serias dificultades para identificar lo relevante en los textos (Echandi, 2018).

La sociedad necesita bibliotecas con recursos digitales. Éstas enfrentan el desafío de reformular los servicios, como la promoción de la lectura (Ramírez Leyva, 2019); en este contexto, las TIC pueden ser el vehículo a prácticas de lectura disfrutables, a socializar con otros grupos así como a planificar estrategias que acerquen a los libros, reafirmando la vocación social de las bibliotecas.

Las bibliotecas públicas son espacios de inclusión que aportan a la construcción de una sociedad lectora ya que fungen como puente entre ciudadanos, lectores potenciales y los libros; por su parte, el bibliotecario dinamiza las colecciones para su uso y también las integra a los demás servicios. Su ejercicio profesional debe estar mediado por la realidad social de manera consciente (Calzada Alvarado, 2006).

Con la aparición de las TIC el bibliotecario requiere nuevas competencias para impulsar la biblioteca a los cambios culturales y sociales (Hernández Pérez y García Moreno, 2010). Deberá adaptar sus modelos tradicionales a esquemas más versátiles; deberá ser un lector para responder de manera efectiva a los requerimientos de usuarios y así potenciar la biblioteca como el espacio cultural que abre el acceso al conocimiento y aporta en la formación de lectores (Cordón García y Gómez Díaz, 2019).

El presente artículo reporta los resultados de una investigación-acción en el marco de la Especialización en Promoción de la Lectura (EPL) de la Universidad Veracruzana (UV), realizada con bibliotecarios públicos. Se advirtió la situación de las bibliotecas en relación con los servicios de promoción de la lectura usando las TIC, con el objetivo de analizar la práctica interna para transformarla y así mejorar los servicios. 
El objetivo fue promover la lectura y la escritura utilizando las TIC en un grupo de bibliotecarios públicos para coadyuvar a la mejora de sus prácticas profesionales, incentivar el uso de recursos digitales, integrarlos como herramienta para el disfrute de la literatura e impulsar redes de colaboración y conocimiento.

La hipótesis que orientó el trabajo es que ello puede ocurrir si a través de un taller de lectura se utilizan las estrategias e instrumentos adecuados al perfil de los participantes, se promueve el uso de herramientas digitales, se alienta el intercambio de experiencias lectoras con bibliotecarios de otros lugares, usando para ello manifestaciones culturales y artísticas relacionadas con la literatura.

En este trabajo se demuestra que el acompañamiento a las tareas de los bibliotecarios así como la capacitación en promoción de la lectura utilizando las TIC tiene un efecto positivo sobre la percepción de la necesidad de un cambio en sus prácticas profesionales considerando la importancia de la lectura en la sociedad.

\section{La lectura en el siglo XXI}

La importancia de la lectura es motivo de reflexión desde diversos sectores sociales y organismos, donde prevalece el consenso de que el tema tiene que trascender el simple discurso.

Aunque en la educación la competencia lectora es fundamental para cualquier profesión y por ende para el desarrollo social y económico, es decir, para la economía del conocimiento, el proceso lector debe asumirse como una práctica social, donde resalta "justamente ese carácter: una forma automatizada, interiorizada y socialmente significativa de hacer algo" (Cruces, 2017: 13). Formar lectores implica la participación de padres, maestros, bibliotecarios y de todo aquél que considere ineludible asumir el compromiso de leer. Esta práctica se inicia con el disfrute del texto, asociado a la dimensión estética, así como a la acción valorativa. El lector incipiente, el leedor, como lo refiere Bartolomé (Argüelles, 2014), necesita entrenamiento y acompañamiento para hacerse lector y quien asuma esta tarea requiere de una formación. Las competencias de comunicación oral y escrita son fundamentales en todo proceso formativo y posteriormente en el desempeño laboral y social; pero además, teniendo en cuenta que la lectura está relacionada con la capacidad de adquirir nuevos conocimientos y despertar un sentido crítico frente al mundo, se convierte en una actividad de poder, no sólo individual cuando el lector construye el sentido por medio de sus propias experiencias y adquiere mejores destrezas para su desempeño social (López Acosta y Jarvio Fernández, 2018), sino 
también colectivo al ponerlas en diálogo con otras personas, pues son el lector y las sociedades lectoras mejores agentes de desarrollo para un país y un gran aporte en el ejercicio de ciudadanía.

Leer hoy adquiere connotaciones diversas. Resalta el carácter interactivo de la lectoescritura multimedia que combina imagen, sonido y texto (Martos Nuñez y Fernández-Fígares, 2013). Los lectores, de ser usuarios y consumidores, han pasado a ser productores y gestores del mundo online, sin importar el espacio, la época o la cultura, donde se comparten experiencias, imágenes, libros, música y conocimiento (Zafra, 2017). "Escribimos para todos. La lectura digital ha permitido que la comunicación se horizontalice en el sentido de que cada persona escribe sobre lo que lee y todos pueden participar. El lector se ha transformado en autor" (Cruces, 2017: 22).

Estos elementos dan un nuevo sentido al concepto del libro, el cual es más social y colaborativo, más descontextualizado y más heterogéneo (Cordón García, 2019), donde se deja de lado la apreciación de una lectura concluída o cerrada como en el caso del libro impreso. "El libro como una suerte de palimpsesto digital susceptible de una permanente renovación, de un crecimiento ininterrumpido, de una contemporaneidad constante" (Cordón García, 2014: 9).

No obstante lo anterior, en México las encuestas de lectura de 2006 (Conaculta, 2006), 2012 (FMFL, 2012) y 2015 (Conaculta, 2015) muestran una exigua actividad de lectura en la población. Se reporta que de 2006 a 2015 el número de libros leídos pasó de 2.9 a 3.5. Y si atendemos los resultados que proporciona el INEGI (2019) a través del Módulo sobre Lectura (MOLEC), se observa un descenso de 10 puntos porcentuales, de 2015 a 2019, en la población mayor de 18 años que establece leer distintos materiales como libros, periódicos, revistas, historietas, tanto en formato impreso como digital. El reto es ineludible, y el papel de la biblioteca fundamental para afrontarlo. Es necesario eliminar la imagen de depósito de libros, sustituir el espacio pasivo a uno activo y altamente estimulante, donde las expresiones culturales sean utilizadas para cumplir nuevos objetivos.

\section{La biblioteca pública y la formación de lectores}

Aunque la promoción de la lectura ha sido consustancial a los servicios de la biblioteca, existen rezagos en cuanto a sus alcances. Es necesario atender la "idea que se tiene sobre el lugar social de la biblioteca pública en la formación de los lectores" (Álvarez et al., 2008: 173), así como la consideración de "los sustentos contextuales, conceptuales, teóricos y metodológicos que tienen esas prácticas" (Álvarez et al., 2008: 165). Además, pocos usuarios la utilizan para leer voluntariamente y por placer, aunque se disponga de acervos de literatura. 
La IFLA amplía la definición de biblioteca pública de la Unesco: se deben integrar servicios derivados del auge de la tecnología. Las bibliotecas públicas, considerando las diferencias por regiones, tienen la oportunidad de revertir la "brecha digital" ofreciendo acceso y capacitación, coadyuvando a combatir el analfabetismo digital (IFLA-Unesco, 2001: 8).

La forma de abordar la promoción de la lectura depende de cada centro. El bibliotecario debe manejar la información frente a la abundancia, por lo que requiere una continua renovación de conocimientos para integrar nuevos roles y nuevas competencias (Alonso Arévalo, 2016). En México se carece de una formación especializada y la capacitación para promover la lectura es empírica y soportada por sesiones esporádicas. El requerimiento actual es mayor: "desarrollar habilidades y conocimientos para leer e interpretar textos pertenecientes a un entorno sociocultural multimodal [...] que conjuga en un mismo plano textual de la lengua escrita, el sonido y la imagen" (Gómez Camacho, 2016: 19), por lo que se debe trabajar el tema de la multialfabetización que considera la formación de lectores, alfabetización digital y desarrollo de habilidades informativas. Cada área integra metodologías especiales que deben asumirse como tareas permanentes.

El presente trabajo se desarrolló en la EPL de la UV, México -realizado por la primera autora bajo la dirección de la segunda-, con bibliotecarios públicos para promover la lectura utilizando las TIC. Se realizó el estudio de las características del grupo, el desarrollo e implementación del plan de intervención y finalmente la estrategia de seguimiento y medición de resultados.

\section{Planteamiento del problema}

En Veracruz existen 513 bibliotecas públicas -10 de Xalapa- coordinadas por la Red Estatal de Bibliotecas Públicas, que a su vez se coordina por una red nacional. La Red Nacional de Bibliotecas Públicas proporciona capacitación sobre promoción de lectura de manera eventual y limitada. La falta de apoyos, continuidad, seguimiento y evaluación de los programas de capacitación son obstáculos supeditados a las administraciones en turno.

De inicio, mediante una encuesta, se conoció la percepción de usuarios sobre las bibliotecas: $90 \%$ dijo que existe deterioro en los espacios, $86 \%$ respondió que los materiales de lectura estaban desactualizados y $90 \%$ que las actividades de promoción de lectura eran nulas. En contraparte, $90 \%$ proponían realizar clubes de lectura considerando temas como el cambio climático, avances tecnológicos, desarrollo social, etc. Entre quienes realizaban algún tipo de lectura, $70 \%$ contestó preferir la literatura y $60 \%$ utiliza los dispositivos electrónicos para leer, 100 \% usa Facebook. 
El proyecto se realizó con 31 bibliotecarios de ocho bibliotecas de la ciudad, la mayoría sin formación profesional en bibliotecología y sólo 25 \% cursó el taller básico de lectura que ofrece la Secretaría de Cultura de México; prevalece la profesión de maestros y la edad oscila entre 26 y 60 años, de estrato socioeconómico medio, y 60 \% son mujeres. El 68 \% carecía del conocimiento sobre las TIC, $61 \%$ lee en formato impreso, $26 \%$ en digital y sólo $13 \%$ en ambos; el dispositivo más usado es el teléfono inteligente y $99 \%$ tiene Facebook, que utiliza para ver fotos y enterarse de noticias. Aunque los bibliotecarios disponen de una plataforma denominada Digitalee -que $86 \%$ conoce- sólo 5 \% dice usarla. El 80 \% manifestó no utilizar la tecnología para promover la lectura y $63 \%$ asume los servicios básicos (préstamo externo, consulta en sala, atención al usuario, referencia y apoyo a tareas) como de promoción de la lectura. Cuentan con tecnología básica que no usan. Tampoco realizan búsquedas de bibliografía especializada sobre el tema, no participan en grupos de lectura, no buscan referencias actualizadas de autores, no tienen redes de colaboración, etcétera.

\section{Metodología}

La población objeto de análisis fueron los 31 bibliotecarios de ocho bibliotecas públicas de la ciudad de Xalapa, Veracruz, y 40 usuarios, seleccionados aleatoriamente de los asistentes a la biblioteca durante una semana. Previo a las sesiones del taller, se aplicaron dos cuestionarios: el primero a bibliotecarios, cuyos resultados permitieron analizar el perfil de cada uno así como conocer el trabajo de las bibliotecas, información con la que se diseñó el programa. El segundo, de tipo exploratorio, se aplicó a los 40 usuarios, con lo que se conoció la opinión sobre servicios, particularmente de actividades de fomento de lectura. Los resultados permitieron elaborar cartografías de cada biblioteca para contribuir a planear los servicios acorde a lo que requieren los usuarios.

Se realizaron 20 sesiones presenciales de dos horas que se implementaron en dos fases. La primera fase consistió en una capacitación sobre herramientas digitales (creación y exploración de blog, redes sociales, búsqueda de información, etc.). La segunda fase integró dos actividades: Ciberlecturas, club de lectura que combinó sesiones presenciales con actividades en Facebook, con duración de un mes; integró obras de dos autores, uno que participó de manera presencial y el otro por Facebook. En la red estuvieron disponibles las lecturas, preguntas y comentarios sobre las sesiones. Esta actividad culminó con el encuentro entre los bibliotecarios y los dos autores. 
Dado que las bibliotecas son "consideradas lugares de encuentro que pueden favorecer la interacción y las actitudes intergrupales mutuas" (Cervantes, Navas y Cuadrado, 2019), la segunda actividad integrada fue Lectores migrantes, con un intercambio epistolar entre bibliotecarios de Xalapa y de Colombia durante un mes para incentivar la lectura y la escritura a través de cartas utilizando lenguaje multimodal con diferentes tópicos culturales. Al final se realizó un video y un blog que incluye los intercambios epistolares (https://lectoresmigrantes.wordpress.com/).

Para el seguimiento de resultados se realizaron entrevistas, registro de video y una bitácora que detalla las actividades implementadas, comentarios y percepciones del grupo. Se consideró la asistencia, participación y apreciaciones sobre lecturas y actividades. Finalmente se aplicó un tercer cuestionario para confrontar los avances en relación a los resultados del primero.

\section{Procedimiento}

\section{Socialización de recursos digitales}

Entre las páginas web utilizas, listadas en la Tabla 1, se exploraron páginas de Facebook de bibliotecas y entidades que promueven y publican contenidos sobre el tema. Se revisaron también artículos relacionados con la formación de lectores.

\begin{tabular}{|l|l|l|}
\hline \multicolumn{1}{|c|}{$\begin{array}{c}\text { Tipo } \\
\text { de plataforma }\end{array}$} & \multicolumn{1}{|c|}{ Nombre } & \multicolumn{1}{c|}{ Descripción y uso } \\
\hline $\begin{array}{l}\text { Bibliotecas } \\
\text { digitales } \\
\text { infantiles y } \\
\text { juveniles }\end{array}$ & $\begin{array}{l}\text { Papalotero } \\
\text { http://papalotero.bnjm. } \\
\text { cu/?secc=737\&bib=in- } \\
\text { fantil }\end{array}$ & $\begin{array}{l}\text { Biblioteca Nacional de Cuba José Martí. Cuentos, fábulas, } \\
\text { poesías, canciones, adivinanzas, trabalenguas, curiosida- } \\
\text { des y manualidades. }\end{array}$ \\
\cline { 2 - 3 } & $\begin{array}{l}\text { TumbleBooks } \\
\text { http://biblioteca- } \\
\text { tumble.com/Default. } \\
\text { aspx?ReturnUrl=\%2f- } \\
\text { ViewByCover.aspx\%3f- } \\
\text { categorylD\%3d66\&ca- } \\
\text { tegorylD=66 }\end{array}$ & $\begin{array}{l}\text { Libros animados con ilustraciones, animaciones, sonido, } \\
\text { música y narraciones (libro electrónico). }\end{array}$ \\
\cline { 2 - 4 } & $\begin{array}{l}\text { Encuentos } \\
\text { https://www.encuentos. } \\
\text { com/infantiles/cuen- } \\
\text { tos-infantiles/ }\end{array}$ & Literatura infantil. \\
\cline { 2 - 4 } & $\begin{array}{l}\text { Maguaré } \\
\text { https://maguare.gov.co/ }\end{array}$ & $\begin{array}{l}\text { Ministerio de Cultura de Colombia. Lecturas, videos, } \\
\text { animaciones y recursos interactivos para niños y jóvenes. }\end{array}$ \\
\hline
\end{tabular}




\begin{tabular}{|c|c|c|}
\hline \multirow[t]{4}{*}{$\begin{array}{l}\text { Plataformas } \\
\text { y bibliotecas } \\
\text { digitales }\end{array}$} & $\begin{array}{l}\text { Bibliotecas de Medellín } \\
\text { http://www.reddebiblio- } \\
\text { tecas.org.co/salalectu- } \\
\text { ra/overdrive-libros-digi- } \\
\text { tales-y-audiolibros }\end{array}$ & Integra información sobre función de bibliotecas. \\
\hline & $\begin{array}{l}\text { Conéctate con la lectura } \\
\text { https://conectatealalec- } \\
\text { tura.cl/ }\end{array}$ & $\begin{array}{l}\text { Noticias, aplicaciones, libros digitales, book trailers, } \\
\text { artículos, etc., útiles para interactuar y usar en la promoción } \\
\text { de la lectura. }\end{array}$ \\
\hline & $\begin{array}{l}\text { Biblioteca Nacional de } \\
\text { Buenos Aires } \\
\text { https://www.bn.gov.ar/ }\end{array}$ & Recursos interactivos de novelas clásicas del país. \\
\hline & $\begin{array}{l}\text { Biblioteca Nacional de } \\
\text { Colombia } \\
\text { http://bibliotecanacio- } \\
\text { nal.gov.co./es-co }\end{array}$ & Recursos interactivos de novelas clásicas del país. \\
\hline \multirow[t]{2}{*}{ Otros } & $\begin{array}{l}\text { Novelas interactivas } \\
\text { http://www.wellness- } \\
\text { losjameos.com/Varios/ } \\
\text { otros/enquienconfias. } \\
\text { html }\end{array}$ & $\begin{array}{l}\text { Permite leer una novela, cambiar su destino e interactuar } \\
\text { con ella. }\end{array}$ \\
\hline & $\begin{array}{l}\text { Digitalee } \\
\text { https://www.digitalee. } \\
\text { mx/quienes-somos/ }\end{array}$ & $\begin{array}{l}\text { Red Nacional de Bibliotecas Públicas de México. Se } \\
\text { accede a novedades para leer desde cualquier dispositivo } \\
\text { conectado a internet. }\end{array}$ \\
\hline
\end{tabular}

Tabla 1. Páginas web, plataformas y recursos digitales

\section{Ciberlecturas, un club de lectura}

Un club de lectura requiere de una metodología así como de planeación previa de selección de textos y estrategias (Moreno Mulas, García Rodríguez y Gómez Díaz, 2017). Se eligieron cuentos de Guevara del Ángel (2006), xalapeña con una narrativa sencilla, y del colombiano Muñoz (2017), que aborda temas cotidianos con un manejo distinto en los tiempos verbales, forma de narración y descripción en los relatos. Es decir, ambos con temas comunes pero distinta estructura literaria. Estas experiencias alentaron comentarios y discusiones en Facebook y en algunas sesiones presenciales. Los cuentos de Guevara del Ángel (2006) utilizados fueron "Perfume de gardenias", "Bambi", "Historia de por qué se les teme a las suegras y de cómo éstas pueden salir burladas" y "Motel amor", tomados del libro Santas madrecitas. La dinámica incluyó discusiones previas sobre los temas, lectura en voz alta, recursos multimedia que integró música, fotografías, pinturas, etcétera.

De Muñoz (2017), los cuentos seleccionados fueron "Una noche precaria", "La mata, la matica", "Pierna obstinada" y "Una carrera especial". El grupo participó en el foro de interacción atendiendo preguntas orientadoras. 
Se generaron dinámicas virtuales como la realización de memes a partir de algún personaje, con selección de música donde los bibliotecarios sugerían enlaces de bandas sonoras.

Para abordar las diferencias de las estructuras narrativas se utilizaron diversas dinámicas. Por ejemplo, en Facebook se realizó un ejercicio de escritura colectiva denominado cadáver exquisito, que iniciaba con el comentario de las primeras tres líneas de uno de los cuentos y cada bibliotecario lo continuaba, construyendo la secuencia del cuento. Al final, se leyó el elaborado y se confrontó con el original.

La última actividad consistió en un encuentro con los autores, presencial con Itzel Guevara del Ángel y virtual con Andrés Mauricio Muñoz, donde se habló sobre los relatos y sobre el oficio del escritor.

\section{Lectores migrantes, un intercambio epistolar}

El contacto intergrupal se realizó en sesiones presenciales con los bibliotecarios de Xalapa y a través de WhatsApp con los de Colombia, utilizando relatos multimodales de diversos autores que motivaran la lectura y escritura. Las cartas se enviaban a través del "cartero cibernético" que manejaba quien orientó la actividad, para dar seguimiento al proceso. Se leyeron las mismas lecturas para tener un tema común que sirviera en la elaboración de las cartas.

El tópico de la cultura se integró en el diseño de las actividades, motivando opiniones relacionadas al país de origen: tradiciones, música, gastronomía, formas de expresión, paisajes, entre otros. Los ejemplos se muestran en la Tabla 2.

\begin{tabular}{|c|c|c|}
\hline Sesión & Tema & Relato \\
\hline 1 & $\begin{array}{l}\text { Los paisajes } \\
\text { y sus gentes }\end{array}$ & $\begin{array}{l}\text { Lectura de carta: La historia de Margarita, quien escribe una carta } \\
\text { al compositor Oscar Chávez sobre la matanza de Tlatelolco y la } \\
\text { respuesta hecha canción del compositor. } \\
\text { Relato: https://www.facebook.com/notes/oscar-ch\%- } \\
\text { C3\%A1vez/flor-roja-y-carta-de-margarita-2-de-octu- } \\
\text { bre/10152634945150379/ } \\
\text { Canción: https://www.youtube.com/watch?v=ytlU7sfK5Dc }\end{array}$ \\
\hline & & $\begin{array}{l}\text { Lectura: “iSea por Dios y venga más!", de Laura Esquivel } \\
\text { Audiocuento: "No oyes ladrar los perros", de Juan Rulfo }\end{array}$ \\
\hline
\end{tabular}




\begin{tabular}{|c|c|c|}
\hline \multirow[b]{2}{*}{2} & \multirow[b]{2}{*}{$\begin{array}{l}\text { Tradiciones } \\
\text { y festejos }\end{array}$} & $\begin{array}{l}\text { Lectura de carta: Carta } 1 \text { de "Memorias por correspondencia", de } \\
\text { Emma Reyes }\end{array}$ \\
\hline & & $\begin{array}{l}\text { Lectura: "Un esqueleto de visita", de Evelio José Rosero } \\
\text { Video: El altar de muertos en México } \\
\text { https://www.letraslibres.com/mexico/cultura/como-mon- } \\
\text { tar-tu-ofrenda-muertos?fbclid=IwAR07gejoTjNDj9crIBEQrepkt- } \\
\text { F_6PxNZnz3MwBICS16JbRk5QdVBaj7enPg\#.W9kHG_23mQA. } \\
\text { facebook } \\
\text { Juego interactivo: Las calaveras literarias. } \\
\text { https://es.wikihow.com/escribir-una-calavera-literaria }\end{array}$ \\
\hline \multirow[b]{2}{*}{3} & \multirow[b]{2}{*}{ La gastronomía } & Lectura de carta: Las cartas eróticas de James Joyce \\
\hline & & $\begin{array}{l}\text { Canción: Las décimas de El puntalito (son jarocho) } \\
\text { Lectura: "El manchamanteles", de Laura Esquivel } \\
\text { Video y lectura de la receta: El pozole }\end{array}$ \\
\hline \multirow[b]{2}{*}{4} & \multirow[b]{2}{*}{ La música } & Lectura de carta: "Bienes comunes", de Susana López Rubio \\
\hline & & $\begin{array}{l}\text { Lectura: "Solución inesperada", de B. Traven } \\
\text { Canción: "María Teresa y Danilo", de Hansen y Raúl } \\
\text { Lectura: "Vuelve", de Kavafis } \\
\text { Canción: "Vuelve", Café con aroma de mujer } \\
\text { Lectura: "Canción", de Nicolás Guillén } \\
\text { Canción: "De qué callada manera”, de Pablo Milanés }\end{array}$ \\
\hline 5 & Los lugares & Lectura: "Tito y Pepita" y "Tito, Pepita y el intruso" de Amalia Low \\
\hline
\end{tabular}

Tabla 2. Temas culturales utilizados en las actividades

Se elaboró un altar de muertos y se escribieron calaveras literarias (tipo de copla típica mexicana). Se leyeron y visualizaron historias sobre tradiciones de cada cultura (Día de Muertos en México o Festival de Luces y Faroles en Colombia); se exploraron sitios sobre gastronomía y música, lo que integró relatos acordes a los temas. Se elaboraron videos de cada ciudad para integrarlos a las cartas además de fotos, enlaces a páginas web y de música.

\section{Presentación de Resultados}

Los resultados de la evaluación inicial indican que leer no es actividad cotidiana preferida, ocupa el cuarto lugar con $38.71 \% ; 74.11 \%$ estableció que escuchar música es la actividad que más realizan. Menos de la mitad lee en formato digital y sólo $2 \%$ promueve la lectura utilizando las TIC; su trabajo se circunscribe a lo básico y tradicional: préstamos externo e interno, visitas guiadas, apoyo a la realización de tareas y proyectos, y algunos servicios de promoción de la lectura (10\%). El 90 \% define la biblioteca como un espacio de conocimiento para apoyo de la escuela. 
La encuesta final mostró que se considera a la biblioteca como espacio para conocimiento, pero $80 \%$ amplió esta visión con la de espacio de encuentro social y cultural, artístico e informativo, que aparte de proporcionar información puede aportar en la recreación promoviendo el gusto por la lectura, utilizando las TIC. Cambió el significado de leer. Al inicio $60 \%$ lo relacionó con "tener más conocimiento" o "aprender a hacer buenos escritos, tener más vocabulario y temas de conversación”, al final 70 \% integró expresiones de gusto o placer. El 98 \% afirmó que el uso de los nuevos dispositivos, con sus aplicaciones y herramientas, es de gran utilidad para la promoción de la lectura y que las integrarán a sus servicios bibliotecarios.

El $80 \%$ manifestó que en las bibliotecas digitales consultadas se encuentra un gran repositorio de información para su trabajo y 87 \% manifestó que desde Facebook, Instagram o Twitter pueden integrar actividades para acercar a usuarios y hacer más visible la biblioteca y sus programas.

El $96 \%$ asistió a todas las sesiones y 98 \% participó de manera activa; hubo una disminución de $10 \%$ en las actividades de los espacios virtuales. En la realización de videos, la mayoría expresó sorpresa sobre las posibilidades que brindan los dispositivos digitales para acercar a los usuarios a la lectura; otra de las actividades que mencionan como gratificante y aleccionadora fue el intercambio epistolar, ya que permitió redes de colaboración para seguir promoviendo la lectura, aunque coincidieron, tanto en Xalapa como en Colombia, sobre las condiciones precarias del suministro de tecnología.

\section{DisCUSIÓN Y CONCLUSIONES}

Dado que el uso de las TIC está cada vez más integrado a la vida cotidiana, es impostergable la utilización de las mismas para el fomento de la lectura y la escritura desde la biblioteca pública. El bibliotecario tiene que acercarse a estos lenguajes que hoy transitan entre la imagen, lo verbal y lo auditivo para tener la capacidad de brindar experiencias de lectura en estos medios. Aunque la realización de talleres de lectura y espacios de conversación en torno al libro no son nuevos, adaptar estas iniciativas al ámbito digital permite ampliar los servicios bibliotecarios para tener más cobertura y participación de los usuarios.

Si bien el grupo no estaba familiarizado con el uso de las TIC y pocos las habían utilizado para la promoción de la lectura, se estableció que después de la capacitación, los bibliotecarios descubrieron el potencial de la red para implementar nuevas actividades. El 90\% consideró que la formación y la continua capacitación facilitarán logros en el trabajo. Una preparación previa 
sobre los materiales a usar y la socialización en las plataformas de las lecturas alientan la discusión sobre las mismas y se enriquece la lectura con diversos puntos de vista.

Con las tecnologías la noción de tiempo y espacio se diluye, las comunicaciones se facilitan y amplían, por lo que el uso de dispositivos y aplicaciones es fundamental para generar redes de intercambio que alienten nuevas formas de colaboración entre bibliotecarios. El intercambio epistolar conjugó un sinfín de estrategias que alentaron nuevas actividades en el quehacer bibliotecario, además de experiencias, que de acuerdo a las características de cada lugar, potencian la tarea de formar lectores.

La lectura multimodal proporcionó, tanto en el club de lectura como en el intercambio epistolar, una mejor comprensión y disfrute de las lecturas y expresión en la escritura. La combinación de estos recursos enriquece los relatos e incide en la creación de nuevos significados y en aprendizajes individuales y colectivos.

Los bibliotecarios funcionarios reconocieron la tarea y asumieron el compromiso y la responsabilidad que tienen en la construcción de ciudadanía y en el aporte a la mejora de la sociedad; sin embargo, muchas bibliotecas están en el abandono y no cuentan con recursos suficientes para potencializar sus servicios.

Brindar servicios de promoción de la lectura haciendo uso de las TIC es una exigencia de la biblioteca pública del siglo XXI, pero requiere de apoyo y atención de los gobiernos para crear transformaciones que contribuyan a formar lectores y ciudadanos informados y conscientes.

La mayoría de los bibliotecarios expresaron que la intervención fue de gran ayuda para crear actividades que replicarán en sus bibliotecas; sin embargo, se enfrentan a dificultades como el acceso limitado o nulo a internet y al mal estado de los equipos de cómputo. Construir discursos que ubican a la lectura como actividad social que determina el desarrollo individual y colectivo obliga a considerar la profesionalización de esta actividad, el reto es de las instituciones educativas. Dinamizar la biblioteca depende del bibliotecario en mayor medida; sin embargo, la definición de la estructura y sustentos depende de instancias superiores. Los resultados muestran una gran necesidad de apoyos sobre todo en lo relacionado al suministro de tecnología y formación de cuadros. 


\section{REFERENCIAS}

Álvarez, Didier, Norfi Yamili Ocampo Molina, Yicel Nayrobis Giraldo Girldo, Luz Marina Guerra Sierra, Liliana Melgar Estrada y Maricela Gómez Vargas. 2008. "La promoción de la lectura en las bibliotecas públicas de Medellín”. Revista Interamericana de Biblioteconomía 1 (31): 161-205.

https://aprendeenlinea.udea.edu.co/revistas/index.php/RIB/article/ view/1923/1582

Alonso Arévalo, Julio. 2016. "La biblioteca en proceso de cambio". BiD: textos universitarios de biblioteconomía y documentación (36). https://doi.org/10.1344/BiD2016.36.12

Argüelles, Juan Domingo. 2014. Historias de lectores y lecturas: los caminos de los que síleen, 2a. ed. México: Océano.

Calzada Alvarado, Abraham. 2006. "La importancia de las bibliotecas en la sociedad". Revista de Bibliotecología La Paz 10 (15): 47-52.

http://www.revistasbolivianas.org.bo/pdf/rb/v10n15/v10n15a07.pdf

Cassany, Daniel. 2006. Tras las lineas. Sobre la lectura contemporánea, 3a. ed. Barcelona: Anagrama.

Cassany, Daniel. 2012. En-línea: leer y escribir en la red, 2a. ed. Barcelona: Anagrama.

Cervantes, Luisa, Marisol Navas e Isabel Cuadrado. 2019. "Contacto intergrupal y actitudes en bibliotecas públicas: un estudio con usuarios marroquíes y españoles en Barcelona y Almería”. Revista Española de Documentación Científica (42) 1. https://doi.org/10.3989/redc.2019.1.1581

Conaculta (Consejo Nacional para la Cultura y las Artes). 2006. "Encuesta Nacional de Lectura”. México.

http://sic.conaculta.gob.mx/ficha.php? table=centrodoc\&table_id=144

Conaculta. 2015. "Encuesta Nacional de Lectura”. México.

https://observatorio.librosmexico.mx/files/en cuesta_nacional_2015.pdf

Cordón García, José Antonio. 2010. "De la lectura ensimismada a la lectura colaborativa: nuevas topologías de la lectura en el entorno digital", en Polisemias visuales, editado por Raquel Gómez Díaz y Agustín Lacruz, 39-84. España: Ediciones Universidad de Salamanca.

Cordón García, José Antonio. 2014. Los componentes estructurales del nuevo ecosistema del libro: editores y bibliotecas, el camino hacia la convergencia. España: Fundación Sánchez Ruipérez-Junta de Castilla y León.

https://fundaciongsr.org/wp-content/uploads/2019/03/Los-componentes-estructurales-del-nuevo-ecosistema-del-libro.pdf

Cordón García, José Antonio. 2019. "La lectura académica en el entorno impreso y digital: contextos, disrupciones y canon”, en De la lectura académica a la lectura estética, 17-34. México: UNAM.

Cordón García, José Antonio, Julio Alonso Arévalo y Raquel Gómez Díaz. 2013. Gutenberg 2.0: la revolución de los libros electrónicos. España: Trea.

Cordón García, José Antonio y Olivia Jarvio Fernández. 2015. "Se está transformando la lectura y la escritura en la era digital?”. Revista Interamericana de Bibliotecología (38) 2: 137-145.

https://www.redalyc.org/articulo.oa?id=179038631005 
Cordón García, José Antonio y Raquel Gómez Díaz. 2019. Lectura, sociedad y redes. Colaboración, visibilidad y recomendación en el ecosistema del libro. España: Marcial Pons.

Cruces, Francisco. 2017. ¿Cómo leemos en la sociedad digital? Lectores, booktubers y prosumidores. Madrid: Ariel/Fundación Telefónica.

Dvetach, Laura. 2012. La construcción del camino lector. Argentina: Comunicarte.

Echandi Ruiz, Ma. del Pilar. 2018. "Millennials en la biblioteca: promoción de la lectura recreativa en el entorno digital”. Cuadernos de Documentación Multimedia (30): $35-58$.

https://www.researchgate.net/publication/330379758_Millennials_en_la_bi-

blioteca_promocion_de_la_lectura_recreativa_en_el_entorno_digital

Freire, Pablo. 1972. Pedagogía del oprimido, 3a. ed. Argentina: Siglo XXI Editores.

Freire, Pablo. 1998. La educación como práctica de la libertad, 3a. ed. México: Siglo XXI Editores.

Ferrada, Donatila y Ramón Flecha. 2008. "El modelo dialógico de la pedagogía: un aporte desde las experiencias de comunidades de aprendizaje”. Revista estudios pedagógicos de Valdivia 1 (XXXIV): 41-69.

https://scielo.conicyt.cl/scielo.php?pid=S071807052008000100003\&script=sci_arttext

FMFL (Fundación Mexicana para el Fomento de la Lectura). 2012. "Encuesta Nacional de Lectura”. México.

http://www.educacionyculturaaz.com/wp-content/uploads/2013/04/ENL_2012.pdf

Gómez Camacho, Alejandro. 2016. La alfabetización multimodal: nuevas formas de leer y de escribir en el entorno digital. España: Síntesis.

Gómez Díaz, Raquel, Araceli García Rodríguez, José Antonio Cordón García y Julio Alonso Arévalo. 2016. Leyendo entre pantallas. España: Trea.

Guevara del Ángel, Itzel. 2006. Santas madrecitas. México: Tierra Adentro.

Hernández Pérez, Tony y María Antonia García Moreno. 2010. "Los retos de la alfabetización informacional en las bibliotecas: guía para superar la brecha entre nativos e inmigrantes digitales". Revista de Ciências e Tecnologias de Informação e Comunicação do CETAC.MEDIA (13): 1-20.

https://e-archivo.uc3m.es/handle/10016/10162

IFLA-Unesco (Federación Internacional de Asociaciones de Bibliotecarios y Bibliotecas-Organización de las Naciones Unidas para la Educación, la Ciencia y la Cultura). 2001. "Directrices IFLA/UNESCO para el desarrollo del servicio de bibliotecas públicas”.

http://hdl.handle.net/10421/344

INEGI (Instituto Nacional de Estadística y Geografía). 2019. "Principales resultados". Módulo sobre Lectura (MOLEC).

https://www.inegi.org.mx/contenidos/programas/molec/doc/resultados_molec_feb19.pdf

Lahire, Bernard. 1995. "Escrituras domésticas. La domesticación de lo doméstico". Revista Lectura y Vida 29 (3): 6-23.

http://www.lecturayvida.fahce.unlp.edu.ar/numeros/a29n3/29_03_Lahire.pdf

López Acosta, Sergio Antonio y Antonia Olivia Jarvio Fernández. 2018. "Promoción de la lectura en espacios virtuales: el caso del Portal Lectores y Lecturas de la Universidad Veracruzana" Revista Álabe 18: 1-18. https://dialnet.unirioja.es/ejemplar/492751 
Martos Nuñez, Eloy y Mar Campos Fernández-Fígares, coord. 2013. Diccionario de nuevas formas de lectura y escritura. España: RIUL-Santillana.

Moreno Mulas, María Antonia, Aracely García Rodríguez y Raquel Gómez Díaz. 2017. "Conversando en la nube: cómo organizar un club de lectura virtual". Revista General de Información y Documentación 27 (1): 177-199. http://revistas.ucm.es/index.php/RGID/article/view/56566

Muñoz, Andrés Mauricio. 2017. Hay días en que estamos idos. Colombia: Planeta.

ONU (Organización de las Naciones Unidas). 2015. Agenda para el desarrollo sostenible 2013. NY: PNUD (Programa de las Naciones Unidas para el Desarrollo).

Parra, Andrés. 2018. La promoción de lectura es un diálogo entre iguales. https://maguared.gov.co/la-promocion-de-lectura-es-un-dialogo-entre-iguales-perfil-de-andres-parra-promotor-de-lectura/

Ramírez Leyva, Elsa. 2019. De la lectura académica a la lectura estética. México: UNAM.

Unesco. 1994. Manifiesto sobre la biblioteca pública. https://unesdoc.unesco.org/ark:/48223/pf0000112122_spa

Zafra, Remedios. 2017. El entusiasmo. Precariedad y trabajo creativo en la era digital. España: Anagrama.

Para citar este texto:

Rendón-Galvis, Sindy Catherine y Antonia Olivia Jarvio-Fernández. 2020. "El uso de las TIC para promover la lectura en bibliotecas públicas con la intervención de los bibliotecarios". Investigación Bibliotecológica: archivonomía, bibliotecología e información 34 (83): 129-144. http://dx.doi.org/10.22201/iibi.24488321xe.2020.83.58095 